
\title{
Reseracth Sulure \\ Flow Characteristic Research of Children Subjected to Obstructive Sleep Apnea-Hypopnea Syndrome
}

Huikun Cai ( $\square$ caihuikun@xmu.edu.cn )

Xiamen University

Chang Xu

Xiamen University

Haoyang Xue

Xiamen University

Yufeng Guo

Children's Hospital of Xiamen

Lijun Su

Xiamen University

Xingqiang Gao

Children's Hospital of Xiamen

\section{Research Article}

Keywords: OSAHS, upper airway, flow characteristic, computational fluid dynamics, 3D printing.

Posted Date: November 2nd, 2021

DOI: https://doi.org/10.21203/rs.3.rs-970492/v1

License: (c) (i) This work is licensed under a Creative Commons Attribution 4.0 International License.

Read Full License 


\title{
Flow Characteristic Research of Children Subjected to Obstructive Sleep Apnea-Hypopnea Syndrome
}

\author{
Huikun Cai ${ }^{a,}$, Chang Xu ${ }^{a}$, Haoyang Xue ${ }^{\mathrm{a}}$, Yufeng Guo ${ }^{\mathrm{b}}$, Lijun $\mathrm{Su}^{\mathrm{a}}$, Xingqiang Gao ${ }^{\mathrm{b}}$ \\ ${ }^{a}$ Department of Mechanical and Electrical Engineering, Xiamen University, Xiamen City, Fujian Province, China, 361102 \\ ${ }^{\mathrm{b}}$ Children's Hospital of Xiamen, Xiamen City, Fujian Province, China, 361006 \\ " Corresponding author. Cai Huikun, Email: caihuikun@xmu.edu.cn; Telephone: 15659269026; Fax: +86-592-2186383; \\ Address: No. 4221-134, Xiangan South Road, Xiangan South District, Xiamen City, Fujian Province, China, 361102
}

\begin{abstract}
Revealing the structural morphology and inner flow field of the upper airway is important for understanding obstructive sleep apnea-hypopnea syndrome (OSAHS) incidence phenomena and pathological diagnosis in children. However, the present study is usually concentrated on adults, but achievement cannot be directly applied to children because of different inducing factors. Therefore, this paper employs flow characteristics and a simulation method for child OSAHS. It is found that the Reynold number changes highly throughout the whole upper airway, and the laminar assumption is no longer suitable for low Reynold number flow, which is much unlike classic fluid mechanics. Turbulent models of Standard k- $\omega$ and Spalart-Allmaras were developed prior to suggestion. The simulation is validated by experiments with an error of approximately $20 \%$. Additionally, carried out in this analysis is the influence of adenoidal hypertrophy with different narrow levels. The cross-sectional area, flow velocity, pressure drop and volume rate will change greatly when the narrow level is above $64 \%$ of the upper airway, which can be a quantitative explanation for medical intervention if adenoid hypertrophy blocks $2 / 3$ of the upper airway in the common clinical judgment of otorhinolaryngology. It is expected that this paper can be a meaningful instruction on OSAHS surgery plan making as well as recovery evaluation postoperatively.
\end{abstract}

KEYWORDS OSAHS, upper airway, flow characteristic, computational fluid dynamics, 3D printing.

\section{Introduction}

Obstructive sleep apnea-hypopnea syndrome (OSAHS) can greatly affect children's physical and psychological health in terms of craniofacial anomalies, cardiovascular disease, retinopathy, lymphoid hyperplasia and maldevelopment [1-3], and its prevalence has increased to $8 \%$ since 2005 [4]. Traditional diagnosis is always based on medical imaging technology, such as computed tomography (CT) and magnetic resonance imaging (MRI), as these medical images can clearly show structural abnormities in the upper airway. However, further pathology mechanism analysis, such as flow and pressure disorders caused by structural abnormities, cannot be gained from medical images or in vitro clinical nasal resistance tests, which can only test nasal pressure but not detailed inner flow status of the upper airway.

Benefiting from the development of computational fluid dynamics (CFD), it can fully capture the flow characteristics of the upper airway in terms of pressure, velocity and mass flow rate so that it is increasingly used in the study of OSAHS. An initial trial was carried out in adults and proved that CFD is a potentially useful modality for the clinical assessment of OSAHS medical properties. In contrast to adults, the main factors inducing OSAHS in children are adenoid and tonsillar hypertrophy but not soft palate overlong or blocked-up [5-7]. Consequently, its upper airway structure, clinical manifestation and therapeutic regimen are also different from those of adults, and the research achievement of adult OSAHS cannot be directly applied to child OSAHS therapy. Therefore, revealing the structural morphology and flow characteristics of the child upper airway has become a new interesting and hot topic, as it is important and meaningful for the understanding of child OSAHS incidence phenomena and pathological diagnosis.

For child OSAHS, Raanan et al. [8-9] studied the differences in upper airway structure with and without OSAHS and found that in children with moderate OSHAS, the upper airway was restricted both by adenoids and tonsils as well as the soft palate. However, they did not show a flowing process or flowing field in the upper airway. Chun et al. [10] used CFD to model the effect of airway geometry on internal pressure in the upper airway of three children with OSAHS and three controls and suggested that pharyngeal airway shape in children with OSHAS significantly affected internal pressure distribution compared to nasal resistance, but the model was not a whole upper airway model, as they did many simplifications on nasal structure that could influence pressure and flow fields. Luo et al. [11] built 
models of the upper airway from the nares to trachea before and after adenotonsillectomy (AT), and CFD results showed that the apnea hypopnea index decrease after AT was strongly correlated with a reduction in the maximum pressure drop in the region where the tonsils and adenoid constricted the pharynx. Zhao et al. [12] analyzed the effect of orthodontic treatment on OSAHS in CFD simulations and found that orthodontic treatment could expand airway volume from the palatopharynx to the glossopharynx, lower negative pressure and flow resistance of narrow areas in the upper airway and had a positive influence on OSAHS. Slaats et al. [13] built a 3D reconstruction from functional respiratory imaging (FRI) combined with CFD, used a linear model to solve the flowing process and found that children with more severe OSA had a smaller volume of the overlap region between the adenoids and tonsils. However, these simulations need more experimental validations.

As mentioned above, research on the upper airway structure, clinical manifestation and therapeutic regimen of child OSAHS has gained many results, but there are still many problems that need solutions by numerical and experimental investigations. One of the problems is that there is no convincing numerical method for inner flow field research. Due to the simplified structure of the upper airway, different CFD models were applied in the simulations and gained different results, some of which disagreed greatly with the other in values or even opposite in tendency. Another reason is that many simulations lack experimental validations to prove model rationality, as traditional validations are limited greatly in terms of accuracy, reliability, simplicity, convenience and operability. Therefore, this paper presents a complete model of the child upper airway starting from the anterior naris and ending at the beginning of the trachea. CFD simulations with different solving models are built and compared and validated by lab experiments using 3D printing technology. It is expected to gain a thorough flow characteristic and pressure field of the upper airway and can be helpful for understanding the pathological mechanism of OSAHS in children.

\section{Methods and theories}

The study was approved by the Ethics Committee of Children's Hospital of Xiamen, China, and all methods were performed in accordance with the relevant guidelines and regulations. Informed consent was obtained from parents of volunteer subjects. As shown in Table 1, control subjects with normal growth and development were matched to subjects with OSAHS by age, sex, ethnicity, weight, and height who had no surgery and no airway abnormalities diseases.

Table 1 Detailed clinical data of children with and without OSAHS.

\begin{tabular}{ccccccc}
\hline Subjects & Sex & Age & Height/cm & Weight/kg & AHI of PSG/pcs/h & LSaO2 of PSG \\
\hline Child with OSAHS & Boy & Six years and one month & 115 & 19 & 27 & $70 \%$ \\
Child without OSAHS & Boy & Six years and five months & 117 & 21 & 2 & $90 \%$ \\
\hline
\end{tabular}

\subsection{Model reconstruction and CFD simulation setting}

A completed upper airway should include the nasal, nasopharynx, oropharynx, laryngopharynx and glottis. Therefore, CT images from the anterior naris to tracheal beginning with a scanning layer spacing of $1.25 \mathrm{~mm}$ were imported into commercial software (Amira 3.0, TGS, Inc.) for a 3D model reconstruction. Airway cross-sections perpendicular to the airway direction were defined at different locations, and thirteen cross-sections are shown in Table 2 and Figure 1. To improve grid quality, Materialise 3-matic software was used to optimize the qualities of triangle slices in the *.stl file. The optimization process was as follows: 1) homogenized triangle slices; 2) built well-flat airway inlet and outlet; 3) refined triangle slices in calculating core area; 4) checked model quality to ensure that there was no overlap, intersection, hole and bad edges existing in triangle slices.

Table 2 Thirteen cross-sections of upper airway.

\begin{tabular}{cccccc}
\hline Cross-section & Location & Cross-section & Location & Cross-section & Location \\
\hline 1 & Anterior naris & 6 & End Inferior turbinate & 11 & Tonsil midsection \\
2 & Nasal limen & 7 & Posterior naris & 12 & Hypopharyngeal beginning \\
3 & $\begin{array}{c}\text { Anterior inferior } \\
\text { turbinate }\end{array}$ & 8 & Nasopharyngeal beginning & 13 & Tracheal beginning \\
& Anterior middle & & & & - \\
4 & turbinate & 9 & Adenoid & & - \\
\hline
\end{tabular}




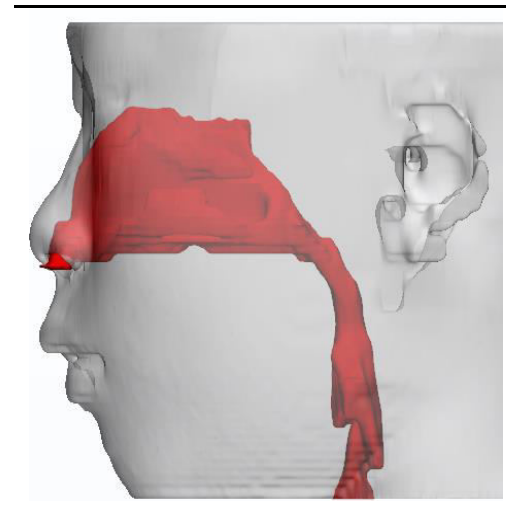

(a)

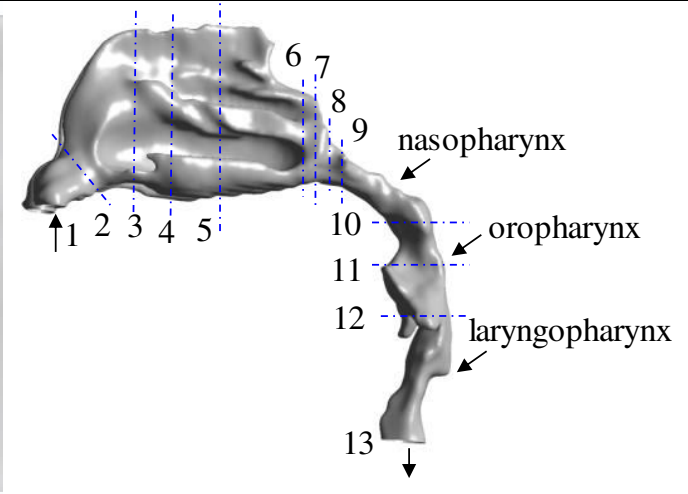

(b)

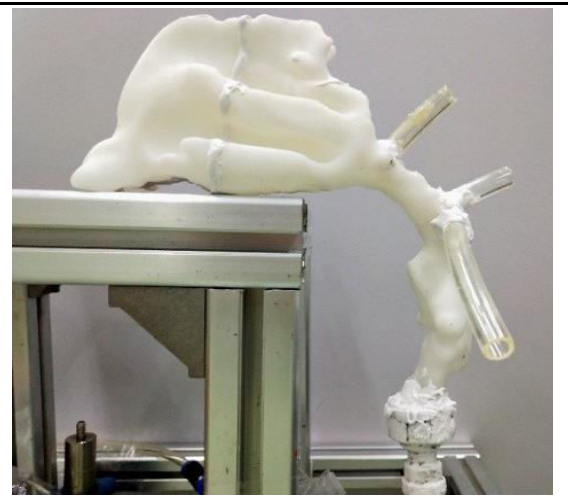

(c)

Figure 1 (a) is a schematic diagram of the upper airway in the body; (b) is the 3D simulation model reconstructed by the CT scanning image; and (c) is the 3D printing model for the lab experiment.

After optimization, the 3D model was imported into commercial CFD meshing software (ICEM, ANSYS, Lebanon, $\mathrm{NH}, \mathrm{USA}$ ) to create unstructured tri/tetrahedral meshes. To capture precise flow characteristics near the wall surface, the mesh in this area was refined with a grid of 10 prismatic layers. The grid height of the first layer should be adjusted to ensure that the $y^{+}$value of the wall surface is small or close to 1 [14]. The model was solved by commercial finite volume CFD software (Fluent, ANSYS, Lebanon, NH, USA). The inlet and outlet both adopted pressure boundary conditions, and inlet pressure was assumed to be zero on inspiration. For continuity and momentum coupling, the solver used the segregated/implicit type and adopted the SIMPLE algorithm to solve the coupled analyses of speed and pressure. The type of discrete equation adopted a second-order upwind scheme. Convergence in all cases was declared only when both strict criteria were satisfied: (a) reduction in all residuals of at least five orders of magnitude; (b) no observable change in surface temperature prediction for an additional tens of iterations.

\subsection{Lab experiment by 3D printing technology}

According to 3D printing analysis [15-17], photosensitive resin was used as the material of the upper airway wall, and stereolithography apparatus technology was adopted in 3D printing. The upper airway model of 3D printing with a wall thickness of $0.2 \mathrm{~mm}$ was of $200 \%$ scale to the simulation model. As shown in Figure 2, a vacuum pump was placed at the outlet of the upper airway (tracheal beginning) as an air exhauster to simulate the inspiration process. The inspiration volume was controlled by the pump working voltage and flow valve placed between the vacuum pump and airway outlet. Two pressure transducers were placed at the nasopharyngeal part (Transducer 1 located in Cross-section 8 and Transducer 2 located between Cross-sections 9 and 10), and Transducer 3 was placed at the oropharyngeal part (Cross-section 10) to measure the pressures of significant points in the upper airway, as most differences of different flow models were concentrated here, and the phenomenon of adverse pressure was also observed in this part according to numerical simulation. The pressure transducer had a measuring range of $-100-100$ $\mathrm{Pa}$ and a measuring precision of $1 \% \mathrm{FS}$. A flowmeter with a measuring range of $0-50 \mathrm{~L} / \mathrm{min}$ and measuring precision of $1.5 \pm 0.2 \%$ FS was used to measure the flow volume rate. Before the experiment, soup suds were smeared on the 3D printing model and junctions to check system air impermeability, and the left nostril was plugged up, similar to the nasal resistance test in vitro.

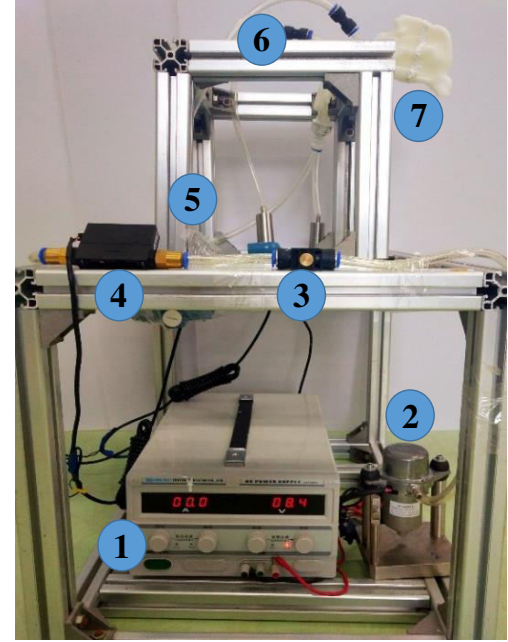

(a)

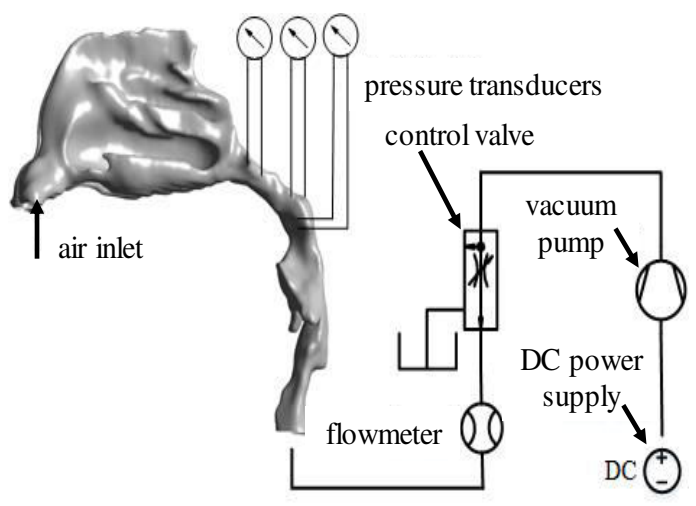

(b) 
Figure 2 Illustration of experimental validation. (a) is the picture of the 3D printing lab experiment, where 1 is the DC power supply, 2 is the vacuum pump, 3 is the control valve, 4 is the flowmeter, 5 is the pressure transducer, 6 is the impulse tube and 7 is the $3 \mathrm{D}$ printing model of the upper airway. (b) Schematic diagram of lab validation by 3D printing.

Dynamic similarity between the 3D printing model and simulation model was maintained. As the 3D printing model was $200 \%$ scale of the simulation model, its characteristic size $D_{3 D}$ was twice that of the simulation model $D_{s i}\left(D_{3 D}\right.$ : $D_{s i}=2: 1$ ). Consequently, to keep the same $R e$, the velocity of 3D printing model $v_{3 D}$ would be half the simulation velocity $v_{s i}\left(v_{3 D}: v_{s i}=1: 2\right)$, referring to formula $R e=\rho v D / \eta$. According to the formula $Q=v \pi d^{2} / 4$, the proportion of flow volume in the 3D printing model to that of the simulation model was 2:1 $\left(Q_{3 D}: Q_{s i}=2: 1\right)$. According to classic fluid mechanics [18], the pressure relationship between the 3D printing model and simulation model was $p_{3 D}=p_{s i}\left(\rho v^{2}\right)_{3 D} /\left(\rho v^{2}\right)_{s i}$, and the pressure of the 3D printing model was quarter that of the simulation model $\left(p_{3 D}: p_{s i}\right.$ $=1: 4)$.

\section{Calculations and results}

\subsection{Reynold number calculation}

As shown in Figure 1, the shapes of thirteen cross-sections of the upper airway are varied and irregular; as a result, their $R e$ values will vary during the whole flowing process. Based on volume flow formula [10]:

$$
\mathrm{Q}=((0.019 * \text { age in years })+0.014) * 1000(\mathrm{~mL} / \mathrm{s})
$$

The flow volume of children aged between 2 and 10 years old ranges from approximately 50 to $250 \mathrm{~mL} / \mathrm{s}$, and Reynold numbers of thirteen cross-sections with flow volume can also be calculated and are shown in Figure 3. For flow volumes of 50 and $100 \mathrm{~mL} / \mathrm{s}$, the Re numbers in thirteen cross-sections are all smaller than 2100 . However, for mass volumes of 200 and $250 \mathrm{~mL} / \mathrm{S}$, the Re numbers can be larger than 2100 beginning in Cross-section 9 due to the area decrease in these cross-sections and increase to the maximum value of 2938 in Cross-section 10 (oropharyngeal beginning) of $250 \mathrm{~mL} / \mathrm{s}$. The average $R e$ values for the whole upper airway of 200 and $250 \mathrm{~mL} / \mathrm{s}$ are approximately 1248 and 1528, respectively. In classic fluid mechanics [18], usually the flow is assumed to be linear if its Reynold number is below 2100, whereas it is turbulent flow if its Reynold number is above 4000 . However, it cannot converge at $200 \mathrm{~mL} / \mathrm{s}$ when the linear model is adopted in the CFD simulation, regardless of whether its average Re number is smaller than 2100. Therefore, numerical analysis of different flow models with different flow volumes is implemented next.

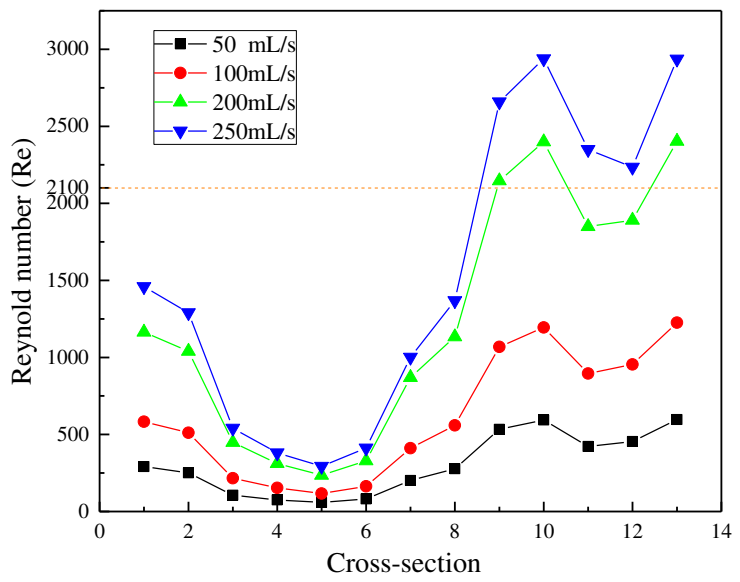

Figure 3 Reynold numbers of thirteen cross-sections vs. different mass flow volumes.

\subsection{Flow distributions of different simulation models}

As shown in Figure 4, it can be seen that air on inspiration from the anterior naris inlet will speed up into the nasal vestibule, and most air will flow through the middle and inferior turbinate after limen nasi. Here, the air decelerates, and after posterior naris, the flow arrives at the nasopharyngeal beginning in terms of linear flow. Since pharyngeal cross-sectional areas decrease, air accelerates quickly and passes through to the outlet. For the laminar flow models in Figure 4 (a) and (b), air flow is linear, and its streamline is regular in most parts of the upper airway. However, due to the sharp cross-sectional area decrease and shape alteration, some turbulence can be observed in the oropharyngeal part at a flow volume of $50 \mathrm{~mL} / \mathrm{s}$, enlarges greatly at $100 \mathrm{~mL} / \mathrm{s}$, and is further aggravated until the simulation cannot converge at $200 \mathrm{~mL} / \mathrm{s}$ using the laminar model. For the turbulent flow model, flow distributions and numerical values are approximately in accordance with the laminar model, but turbulence is clearer in the oropharyngeal part even at flow volumes of 50 and $100 \mathrm{~mL} / \mathrm{s}$, although their Re numbers in the whole flowing process are all smaller than 2100 . 
Therefore, a turbulent CFD model is more suggested for flow characteristic research of the upper airway. If a laminar model is considered, its flow volume should be lower than $100 \mathrm{~mL} / \mathrm{s}$ with an average $R e$ number of 619 . The laminar model is regarded as improper when the flow volume is up to $200 \mathrm{~mL} / \mathrm{s}$ with an average Re number of 1248, which is much unlike the situation in classic fluid mechanics.

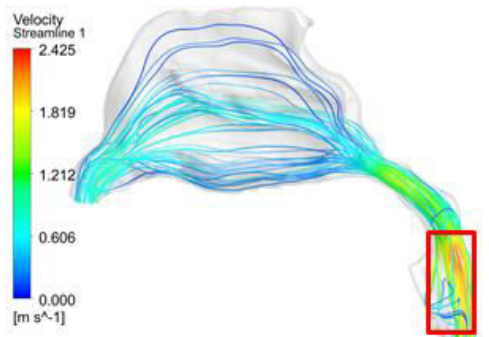

Laminar

(a)

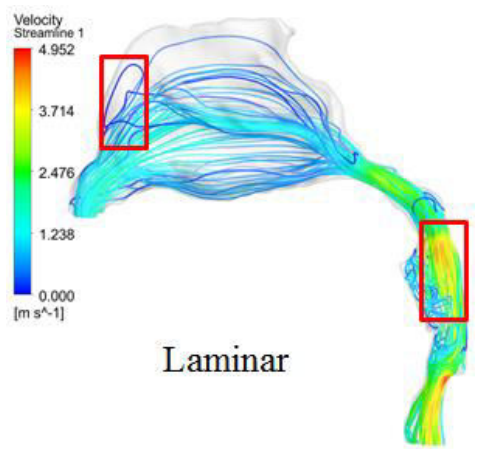

(b)

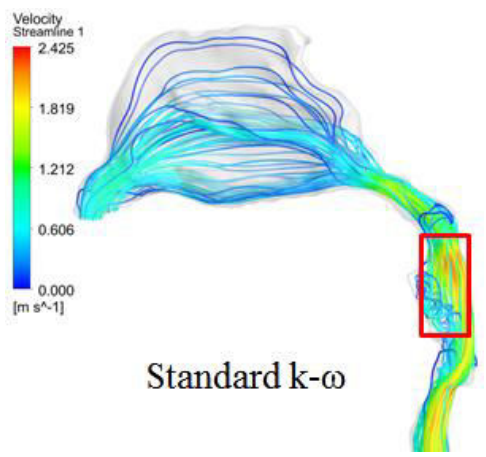

(c)

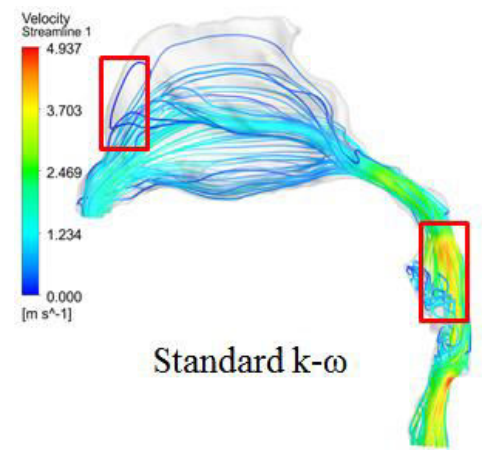

(d)

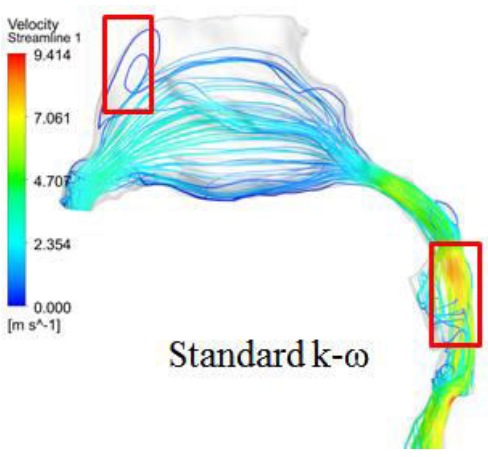

(e)

Figure 4 Flow distributions by different simulation models in the upper airway: (a) and (b) are laminar flow models with flow volumes of 50 and $100 \mathrm{~mL} / \mathrm{s}$, respectively; (c), (d) and (e) are turbulent models adopting the standard k- $\omega$ style with flow volumes of 50, 100 and 200 $\mathrm{mL} / \mathrm{s}$, respectively.

\subsection{Pressure distributions of different simulation models}

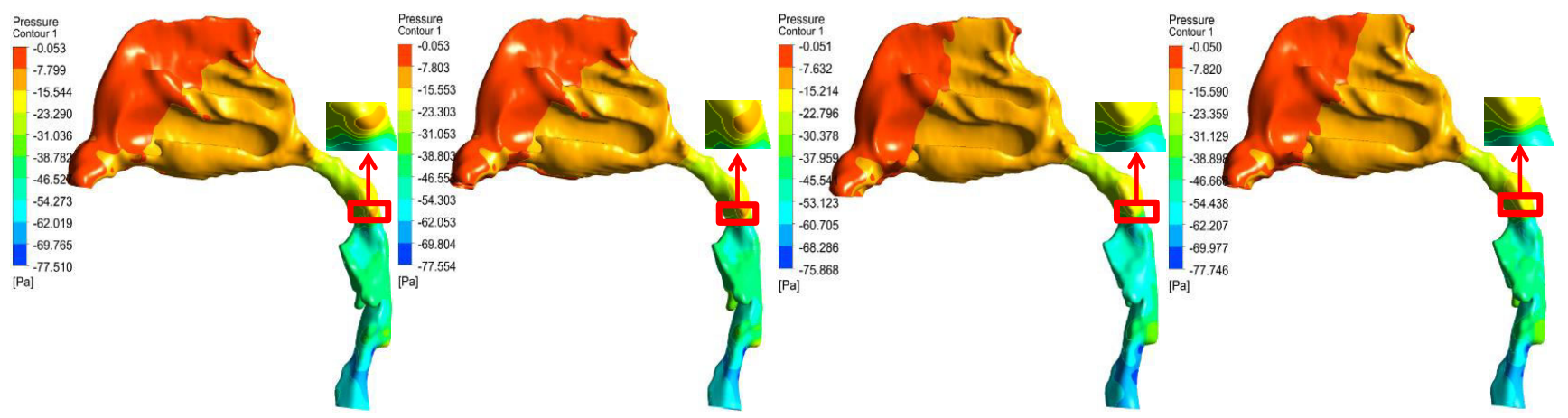

(a) Standard k- $\omega$

(b) Spalart-Allmaras

(c) Standard k- $\varepsilon$

(d) RNG k- $\varepsilon$

Figure 5 Pressure distributions of different turbulent flow models at a flow volume of $200 \mathrm{~mL} / \mathrm{s}$.

As shown in Figure 5, the pressure distributions of different turbulent flow models at a flow volume of $200 \mathrm{~mL} / \mathrm{s}$ are almost the same in terms of the pressure changing tendency and pressure drop value. The difference among the four simulation analyses is that adverse pressure can be clearly observed in the oropharynx when CFD turbulent methods are Standard k- $\omega$ and Spalart-Allmaras, whereas litter or even no adverse pressure occurs in Standard k- $\varepsilon$ and RNG $\mathrm{k}-\varepsilon$ styles. The reason for adverse pressure in the oropharynx is that there is a slight physiological expansion at the oropharyngeal beginning, and consequently, velocity decreases and pressure increases. In classic fluid mechanics, the standard k- $\omega$ and Spalart-Allmaras models are better at simulating air flows of adverse pressure gradients, viscous near-wall regions and boundary layers, especially in low Reynold number flows. In contrast, the standard k- $\varepsilon$ model is weak in handling complex flows with strong pressure gradients and is more suitable for high Reynolds number flows. Although RNG k- $\varepsilon$ provides an analytical formula for flow viscosity of low Reynolds number, it is usually preferred in separate flow and secondary flow. Therefore, as the flow in the upper airway subsizes the physiological structure, 
polytropic cross-section and low Reynolds number, standard k- $\omega$ and Spalart-Allmaras models are suggested when numerical analysis adopts the turbulent CFD method for flow characteristic research of the upper airway.

\subsection{Experimental validation and comparison with simulation}

The adverse pressures at three flow volumes are also detected in lab experiments by 3D printing technology. As shown in Figure 6(c), when the flow volume was $200 \mathrm{~mL} / \mathrm{s}$ in the lab experiment, the oropharyngeal pressure measured by Transducer 3 was $-18.1 \mathrm{~Pa}$, larger than nasopharyngeal pressure of $-22.3 \mathrm{~Pa}$ (measured by Transducers 2). The adverse pressure difference is $4.2 \mathrm{~Pa}$, which is approximately $18.9 \%$ of nasopharyngeal pressure. Correspondingly, in the simulation, the two pressures are $-14.2 \mathrm{~Pa}$ and $-20.5 \mathrm{~Pa}$, respectively, and adverse pressure difference is $6.3 \mathrm{~Pa}$, which is approximately $30.7 \%$ of nasopharyngeal pressure. The same phenomena occurred at flow volumes of 50 and $100 \mathrm{~mL} / \mathrm{s}$. The error between the corresponding laboratory experiment and simulation result of different flow models at different flow volumes is approximately $20 \%$, which can be caused by two aspects: (1) model reconstruction error from CT scanning images; (2) analysis neglecting the effect of some physiological structures, such as vibrissa resistance and neighboring soft tissue interactions. As the reliability and accuracy of laboratory experiments by 3D printing technology have been validated by real in vitro nasal resistance tests in our previous work, adverse pressure experiments can be regarded as reliable. Therefore, adverse pressure cannot be ignored because it can more precisely reflect real air flow in the upper airway.

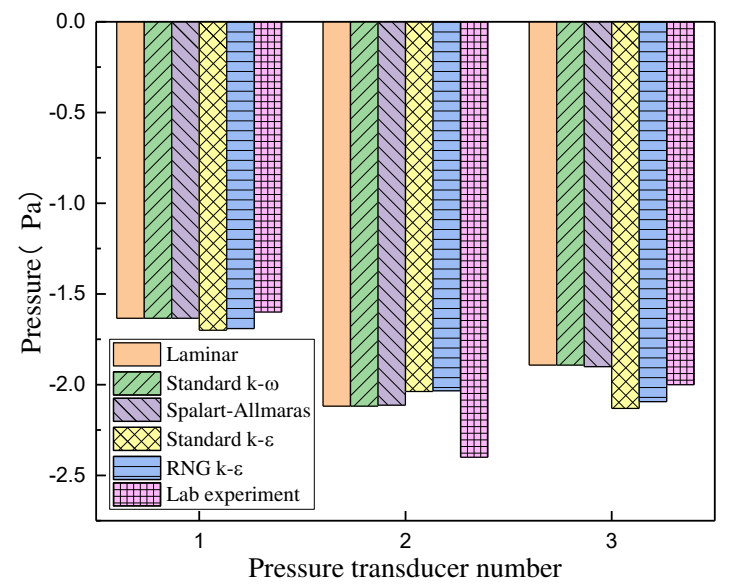

(a) Flow volume of $50 \mathrm{~mL} / \mathrm{s}$

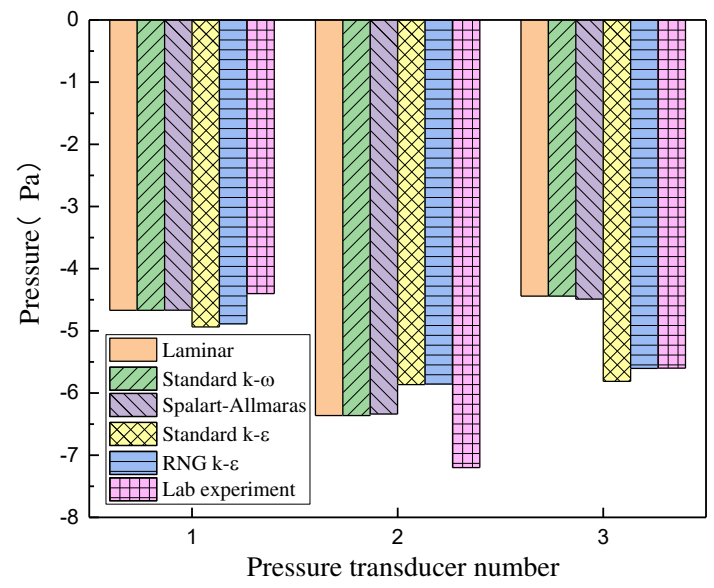

(b) Flow volume of $100 \mathrm{~mL} / \mathrm{s}$

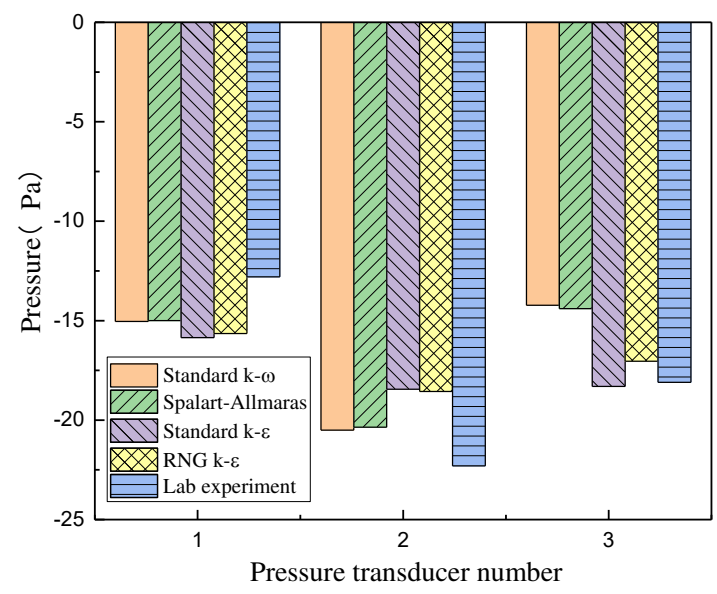

(c) Flow volume of $200 \mathrm{~mL} / \mathrm{s}$

Figure 6 Comparisons of adverse pressure in CFD simulation and lab experiment.

\section{Further studies and discussions on adenoidal hypertrophy}

\subsection{Model reconstruction with adenoidal hypertrophy}

Import CT image of the child with OSAHS described in Table 1 into Amira software, make a virtual operation by 1 $\mathrm{mm}$ issue resection on adenoid hypertrophy each time, and reconstruct eight upper airway models with different 
cross-section areas of adenoid (Cross-section 9) as shown in Figure 7 and Table 3. Define a narrow coefficient as the index of different levels of adenoidal hypertrophy, which is shown as:

$$
\mathrm{NC}=\left(1-\frac{A_{i}}{A_{0}}\right)
$$

where $A_{i}$ is the area of cross-section 9 with different levels of adenoidal hypertrophy, and $A_{0}$ is the area of cross-section 9 without adenoidal hypertrophy. Therefore, $N C$-31 represents that its adenoidal area of cross-section 9 is $31 \%$ of the area without adenoidal hypertrophy, and the number increases, the level of adenoidal hypertrophy is more serious. There is a clinical common otorhinolaryngology finding that if $N C$ arrives at approximately $66.7 \%$, the child will be identified as having pathological adenoid hypertrophy and meet an adenoidectomy criterion. For the child with OSAHS (Figure 7f), his adenoidal area was approximately $12.0 \mathrm{~mm}^{2}$, and his $N C$ was $89 \%$, which means that this child had a severe illness.

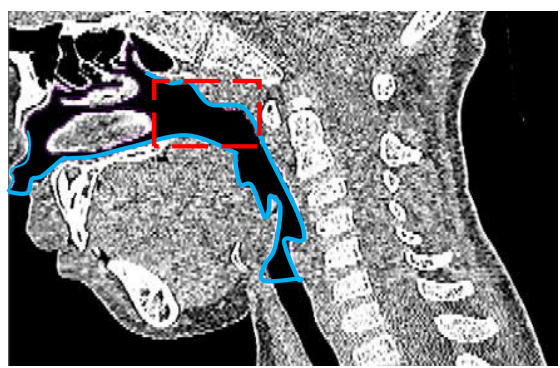

(a) $\mathrm{NC}-0$

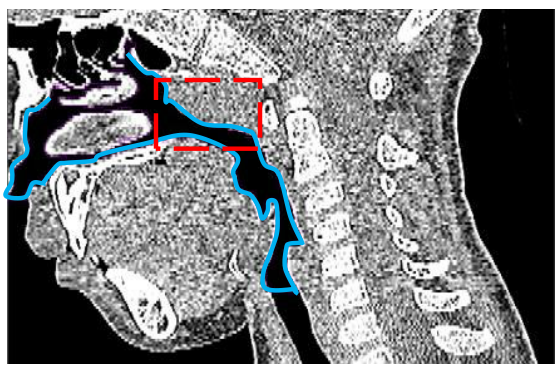

(d) NC-77

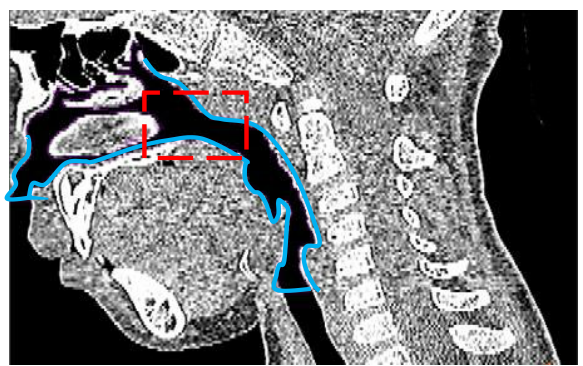

(b) NC-55

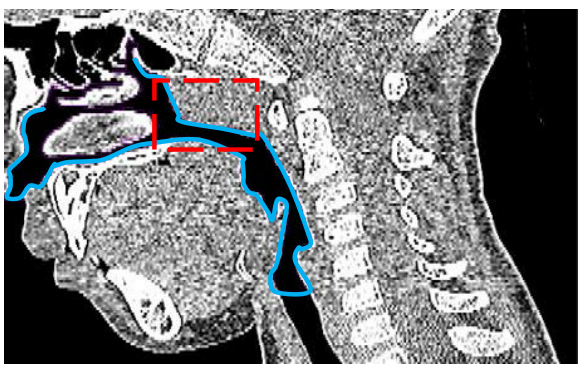

(e) NC-81

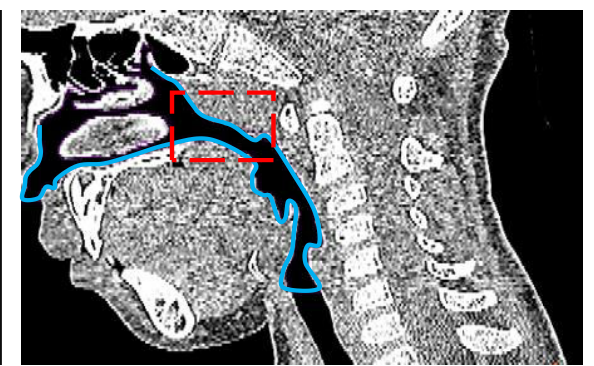

(c) NC-64

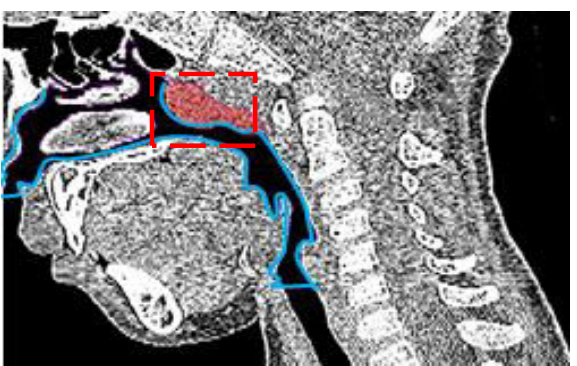

(f) NC-89

Figure 7 Illustration of different levels of adenoidal hypertrophy, where red wireframe represents adenoidal part, light blue wireframe represents upper airway, and light red shaded part in CT image (f) represents adenoidal hypertrophy.

Table 3 Flow characteristics of different levels of adenoidal hypertrophy.

\begin{tabular}{ccccccc}
\hline $\begin{array}{c}\text { Narrow coefficient } \\
N C\end{array}$ & $\begin{array}{c}\text { Flow velocity } \\
v(\mathrm{~m} / \mathrm{s})\end{array}$ & $\begin{array}{c}\text { Cross-section area } \\
\left.10^{-6} \mathrm{~m}^{2}\right)\end{array}$ & $\begin{array}{c}\text { Pressure drop } \Delta p \\
(\mathrm{~Pa})\end{array}$ & $\begin{array}{c}\text { Volume flow rate } Q \\
(\times \mathrm{mL} / \mathrm{s})\end{array}$ & $\begin{array}{c}\text { Velocity } \\
\text { coefficient } C_{v}\end{array}$ & $\begin{array}{c}\text { Flow discharge } \\
\text { coefficient } C_{d}\end{array}$ \\
\hline 0 & 0.83 & 104.8 & 3.60 & 77.81 & 0.351 & 0.314 \\
$31 \%$ & 1.17 & 72.0 & 4.09 & 76.39 & 0.465 & 0.421 \\
$48 \%$ & 1.56 & 54.2 & 4.89 & 76.13 & 0.567 & 0.510 \\
$55 \%$ & 1.77 & 47.2 & 5.44 & 74.85 & 0.609 & 0.546 \\
$64 \%$ & 2.14 & 37.7 & 6.37 & 68.54 & 0.681 & 0.578 \\
$77 \%$ & 3.04 & 23.5 & 10.25 & 67.97 & 0.763 & 0.725 \\
$81 \%$ & 3.40 & 19.6 & 11.78 & 63.08 & 0.796 & 0.753 \\
$89 \%$ & 4.20 & 12.0 & 16.46 & 47.06 & & 0.831 \\
\hline
\end{tabular}

\subsection{Flow and pressure distributions with adenoidal hypertrophy}

As shown in Figure 8 (a-c) and Table 3, when $N C$ is small, air flow gradually accelerates from the anterior naris inlet (cross-section 1) and reaches the largest speed at the oropharyngeal part (between cross-sections 10 and 12). The whole stream line is almost regular and smooth. Until $N C$ reaches $64 \%$, the air speed at the nasopharyngeal part (between cross-sections 8 and 10) increases sharply with an increase of $0.082 \mathrm{~m} / \mathrm{s}$, corresponding to an $N C$ increase of $1 \%$, which is approximately 4.8 times that when $N C$ is below $55 \%$. In addition, the stream line also becomes disordered, and separate flows and large vortices begin to appear. Especially when $N C$ exceeds $80 \%$, the air speed at the adenoid (cross-section 9) becomes the largest in the upper airway, and a high-speed jet flow gradually forms. As a result, this jet flow will produce a strong impact on the airway wall and cause high-frequency flutter on neighboring soft issues in clinical syndromes, which will result in snoring, hypopnea and even sleep apnea. 
The pressure distributions in Figure 8 (d-f) and Table 3 again prove the syndrome. When $N C$ is up to $64 \%$, the air pressure at the adenoid (cross-section 9) decreases sharply with a decrease of $0.427 \mathrm{~Pa}$, corresponding to an $N C$ increase of $1 \%$, which is approximately 14 times that when $N C$ is below $55 \%$. Especially when $N C$ is $89 \%$, its pressure drop between Cross-sections 8 and 9 is approximately $13.8 \mathrm{~Pa}$, which is $69.2 \%$ of the whole pressure drop from inlet to outlet. The high pressure drop in the adenoidal part will strongly absorb neighboring soft issues and consequently induce issue shrinkage or even collapse, which will aggravate clinical syndrome.

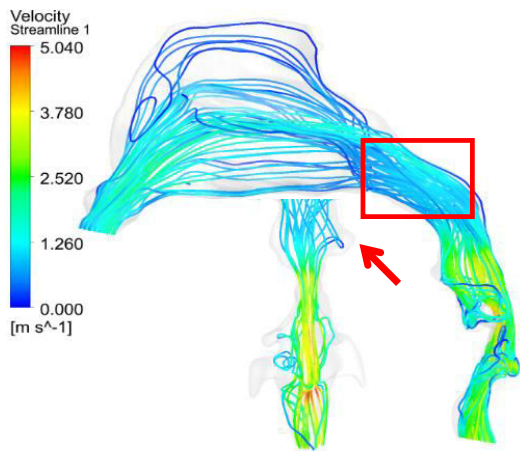

(a) $\mathrm{NC}-0$

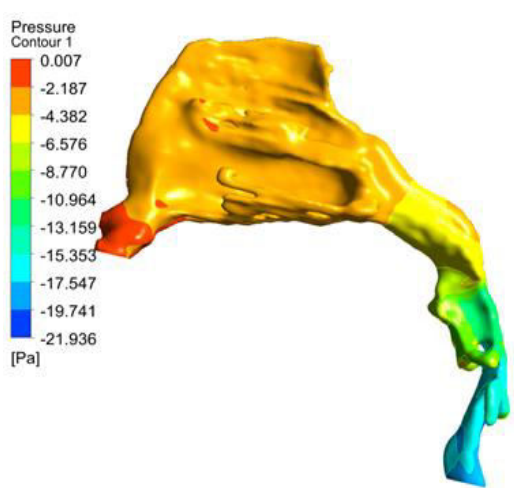

(d) $\mathrm{NC}-0$

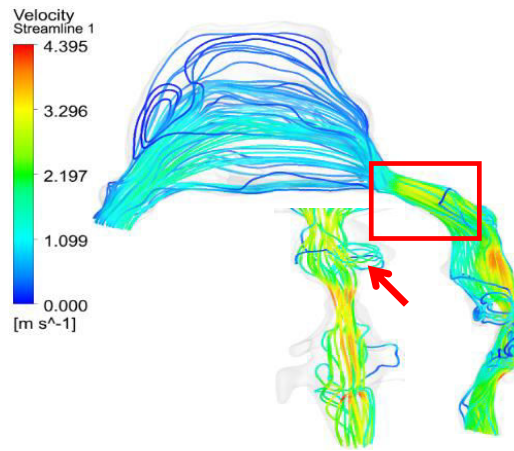

(b) NC-64

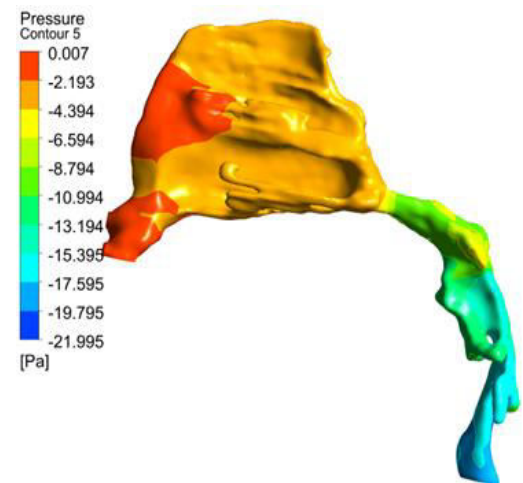

(e) NC-64

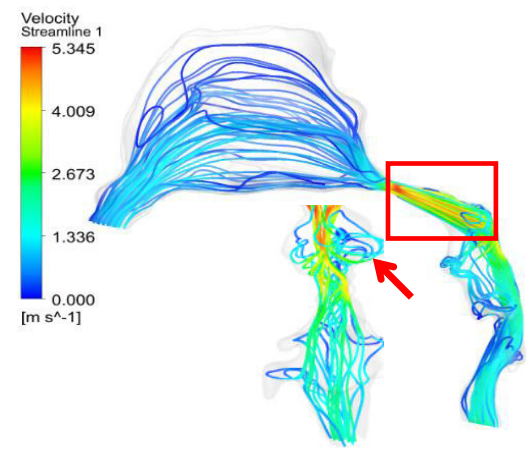

(c) NC-89

Figure 8 Flow distribution (a-c) and pressure distribution (d-f) of different adenoidal hypertrophies.

\subsection{Flow characteristic analysis of upper airway}

Because the cross-sectional area decreases and the pressure drop enlarges, the volume flow rate will decrease with increasing NC. As shown in Table 3, the flow volume initially decreases slowly and remains at a level of approximately $76 \mathrm{~mL} / \mathrm{s}$ when $N C$ is below $64 \%$. Once $N C$ reaches $64 \%$, the flow volume decreases quickly from $74.85 \mathrm{~mL} / \mathrm{s}$ at $55 \%$ to $68.54 \mathrm{~mL} / \mathrm{s}$ and drops to $47.06 \mathrm{~mL} / \mathrm{s}$ until the block reaches $89 \%$. Medically, for a child with adenoidal hypertrophy, there is a qualitative judgment that if adenoid hypertrophy blocks $2 / 3$ of the upper airway, it should be a medical intervention. The sharp decrease in flow volume above $64 \% N C$ can be a quantitative explanation for this operation index and is a very useful and instructive principle for OSAHS mechanism and operation scheme enactment in clinical medicine.

Additionally, gained from the study are flow characteristic parameters: velocity coefficient and flow discharge coefficient. Note that the cross-sectional area of the adenoid (cross-section 9) decreases quickly in the upper airway; it can be assumed to be a throttling orifice, and its flow velocity $v$ and flow volume $Q$ can be calculated as:

$$
\begin{gathered}
\mathrm{v}=C_{v} \sqrt{\frac{2 \Delta p}{\rho}}(\mathrm{m} / \mathrm{s}) \\
\mathrm{Q}=C_{d} A_{i} \sqrt{\frac{2 \Delta p}{\rho}}\left(\mathrm{m}^{3} / \mathrm{s}\right)
\end{gathered}
$$

where $C_{v}$ is the velocity coefficient, $C_{d}$ is the flow discharge coefficient, $A_{i}$ is the adenoid area of cross-section 9 , $\Delta p$ is the pressure drop of cross-section 9 to cross-section 1 and $\rho$ is the air density of $1.29 \mathrm{~kg} / \mathrm{m}^{3}$. From Figure 9 and Table 3, it can be seen that with a narrow coefficient increase, the velocity coefficient and flow discharge coefficient both increase. Their relationships can be expressed by curve fitting equations:

$$
C_{v}=0.3459+0.3522 N C+0.2365 N C^{2}
$$




$$
C_{d}=0.314+0.2272 N C+0.358 N C^{2}
$$

In classic fluid mechanics, the flow discharge coefficient of the throttling orifice remains at a value of 0.5-0.6 if the flow is a full contraction flow, and if not, the coefficient will change with the Reynold number. Therefore, it can be concluded that upper airway flow is an incomplete contraction flow, and the structure of the upper airway plays a guiding role in air flow.

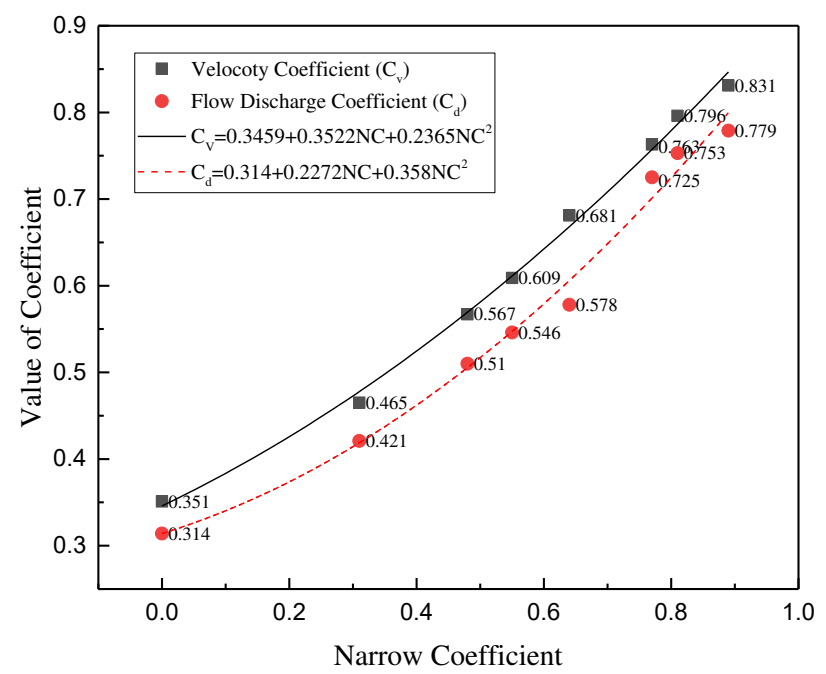

Figure 9 Velocity coefficient $\left(C_{v}\right)$ and flow discharge coefficient $\left(C_{d}\right)$ vs. narrow coefficient $(N C)$

\section{Conclusions}

This paper concentrates on the simulation method and flow characteristics of the upper airway for children with OSAHS. A complete 3D model of the upper airway starting from the anterior naris and ending at the trachea beginning is reconstructed based on CT images and solved in CFD to reveal its flow and pressure distributions. The $R e$ number changes highly in the whole upper airway due to great cross-sectional area and shape alterations. As a result, turbulence can be clearly observed even at a low flow of $100 \mathrm{~mL} / \mathrm{s}$ on inspiration with an average $R e$ of 619 , and the laminar assumption is no longer suitable for flow with a volume rate of $200 \mathrm{~mL} / \mathrm{s}$ and an average $R e$ number of 1248 , as it cannot converge in this status, which is much unlike classic fluid mechanics. In addition, since the turbulent standard k- $\varepsilon$ model and RNG k- $\varepsilon$ model cannot simulate adverse pressure in the oropharynx, turbulent models of standard k- $\omega$ and Spalart-Allmaras are prior to suggestions for upper airway research. The pressure drop and adverse pressure of different flow models and volume rates in the simulation are validated in lab experiments by $3 \mathrm{D}$ printing technology with an error of approximately $20 \%$. Additionally, carried out in this analysis is the influence of adenoidal hypertrophy with different $N C$ levels. When $N C$ is above $64 \%$, the inner flow field will change greatly at the nasopharyngeal part with the appearance of strong turbulence, formation of high-speed jet flow and high pressure drop, and consequently strong absorption on the airway wall and high-frequency flutter on neighboring soft issues, which will result in snoring, hypopnea and even sleep apnea in clinical syndrome. The flow volume will decrease quickly from $74.85 \mathrm{~mL} / \mathrm{s}$ at $55 \% \mathrm{NC}$ to $68.54 \mathrm{~mL} / \mathrm{s}$ at $64 \% \mathrm{NC}$ and drop to $47.06 \mathrm{~mL} / \mathrm{s}$ at $89 \% \mathrm{NC}$, which can be a quantitative explanation for medical intervention if adenoid hypertrophy blocks $2 / 3$ of the upper airway in the clinical common judgment of otorhinolaryngology. It is expected that this paper can provide a further understanding of the OSAHS pathology mechanism and meaningful instruction on surgery plan making as well as recovery evaluation postoperatively.

\section{References}

1. Sun, Y. Investigation of the physical growth and neuropsychological characters in children with obstructive sleep apnea hypopnea syndrome. 2008, Master Dissertation of Qingdao University. (in Chinese).

2. Duan, X., Zheng, H. J. Obstructive sleep apnea hypopnea syndrome study on pathogenesis and prognosis, Journal of Clinical Otorhinolarynglolgy. Head and Neck Surgery, 2017, 31(17), 1376-1380. (in Chinese).

3. Huang, G.-J. Aging effect on sleepiness and apneas severity in patients with obstructive sleep apnea syndrome. European Archives of Oto-Rhino-Laryngology, 2019, 276(12), 3557-3558.

4. Guilleminault, C., Lee, J. H., \& Chan, A. Pediatric Obstructive Sleep Apnea Syndrome. JAMA Pediatrics, 2005, 159(8), 775-785.

5. $\mathrm{Su}, \mathrm{X}$., et al., The evaluation of 128 slice spiral CT for soft palate, hard palate and mandible in patients with obstructive sleep apnea hypopnea syndrom. Journal of Clinical Otorhinolaryngology, Head, and Neck Surgery, 2015, 29(19), 1723-1726. (in Chinese)

6. Ye, J. Y., Li, J. J. Otolaryngologist's perspective in child hood adenotonsillar hypertrophy. Journal of Clinical Otorhinolarynglolgy, 2016, 30(6), 449-154. (in Chinese). 
7. Shen, L., Lin, Z., Lin, X., \& Yang, Z. Risk factors associated with obstructive sleep apnea-hypopnea syndrome in Chinese children: A single center retrospective case-control study. PLOS ONE, 2018, 13(9), 1-16.

8. Arens, R., et al., Magnetic resonance imaging of the upper airway structure of children with obstructive sleep apnea syndrome. American Journal of Respiratory and Critical Care Medicine, 2001, 164(4), 698-703.

9. Arens, R., et al., Changes in upper airway size during tidal breathing in children with obstructive sleep apnea syndrome. American Journal of Respiratory and Critical Care Medicine, 2005, 171(11), 1298-1304.

10. $\mathrm{Xu}, \mathrm{C}$., et al., Computational fluid dynamics modeling of the upper airway of children with obstructive sleep apnea syndrome in steady flow. Journal of Biomechanics, 2006, 39(11), 2043-2054.

11. Luo, H., et al., Computational fluid dynamics endpoints for assessment of adenotonsillectomy outcome in obese children with obstructive sleep apnea syndrome. Journal of Biomechanics, 2014, 47(10), 2498-2503.

12. Zhao, X. N. Of children with OSAHS after Orthodontics treatment airway flow field numerical simulation and characteristic analysis. Master Dissertation of Qingdao University, 2017. (in Chinese).

13. Slaats, M., et al., Predicting the effect of treatment in pediatric OSA by clinical examination and functional respiratory imaging. Pediatric Pulmonology, 2017, 52(6), 799-805.

14. Li, C., Jiang, J., Dong, H., \& Zhao, K. Computational modeling and validation of human nasal airflow under various breathing conditions. Journal of Biomechanics, 2017, 64, 59-68.

15. Song, X. N., Yan, B., Zhou L. L. Application of 3D printing technology in the field of CT technology. CT Theory and Applications, 2015, 24(1), 57-68. (in Chinese).

16. $\mathrm{Su}, \mathrm{L}$. J. Computational fluid dynamics analysis and experimental study of the upper airway of children. Master Dissertation of Xiamen University, 2017. (in Chinese).

17. Yan, Q., Dong, H., Su, J., Han, J., Song, B., Wei, Q., \& Shi, Y. A Review of 3D Printing Technology for Medical Applications. Engineering, 2018, 4(5), 729-742.

18. Jing, S. R., Zhang. M. Y. (2001). Fluid mechanics. Xi'an: Xi'an Jiaotong University Press. (in Chinese).

\section{Acknowledgment}

The presented work was supported by the National Natural Science Foundation of China (Grant No. 52076183), Health Education Joint Project of Fujian Province of China (Grant No. 2019-WJ-33), and Natural Science Foundation of Fujian Province of China (Grant No. 2018D0018).

\section{Authors' contributions}

Huikun Cai contributed to the conception of the study, analysis of results, manuscript preparation and wrote the manuscript;

Chang $\mathrm{Xu}$ and Haoyang Xue performed the simulation and experiment;

Yufeng Guo and Lijun Su helped perform the data analyses and manuscript preparation;

Xingqiang Gao helped perform the analysis with constructive discussions.

\section{Compliance with ethics guidelines}

All authors (the name of author) declare that they have no conflicts of interest or financial conflicts to disclose. 